\title{
Exponential Stability of Coupled Systems on Networks with Mixed Delays and Reaction-Diffusion Terms
}

\author{
Wenxue Li, ${ }^{1,2}$ Tianrui Chen, ${ }^{1}$ and Ke Wang ${ }^{1}$ \\ ${ }^{1}$ Department of Mathematics, Harbin Institute of Technology (Weihai), Weihai 264209, China \\ ${ }^{2}$ Department of Electrical Engineering, Harbin Institute of Technology, Harbin 150001, China \\ Correspondence should be addressed to Wenxue Li; wenxuetg@hitwh.edu.cn
}

Received 23 December 2013; Accepted 20 February 2014; Published 26 March 2014

Academic Editor: Daoyi Xu

Copyright (c) 2014 Wenxue Li et al. This is an open access article distributed under the Creative Commons Attribution License, which permits unrestricted use, distribution, and reproduction in any medium, provided the original work is properly cited.

\begin{abstract}
This paper is concerned with the stability analysis issue for coupled systems on networks with mixed delays and reaction-diffusion terms (CSNMRs). By employing Lyapunov method and Kirchhoff's Theorem in graph theory, a systematic method is proposed to guarantee exponential stability of CSNMRs. Two different kinds of sufficient criteria are derived in the form of Lyapunov function and coefficients of the system, respectively. Finally, a numerical example is given to show the effectiveness of the proposed criteria.
\end{abstract}

\section{Introduction}

Coupled systems on networks (CSNs) have important practical significance; for example, neural networks can be seen as a coupled system, so that they are widely used in physics, biology, and engineering fields [1-4]. In recent years, CSNs have been an active research topic and received considerable attention. When a coupled system is constructed, some unknown small disturbance force, which is unavoidable and affects every movement of the system, has not been taken into consideration. So, whether the coupled system is stable will be an important issue. As a main property of CSNs, stability has been an enduring topic, and many results have been reported [5-7]. Gan and Xu [8] investigated the stability of a delayed reaction-diffusion neural network. The stability problem for a class of neutral-type neural networks was investigated in the paper [9]. Hu et al. [10] considered globally exponential stability for delayed neural networks.

On the other hand, time delay is inevitably in practice because of finite transmission of interaction [11]. Meanwhile, distributed time delays also should be included in the model due to the fact that there may exist a distribution of propagation delays over a period of time in some cases [7, 12-14]. Therefore, both time-varying delay and the distributed timevarying delay should be taken into account when we model CSNs. In addition, to describe the dynamics changes of CSNs more accurately, diffusion effects cannot be avoided (see [1517] and the references therein). As previously shown, the stability of coupled systems on networks with mixed delays and reaction-diffusion terms (CSNMRs) has been a research focus.

The main method that contributes to investigating stability of a system is Lyapunov function. In the literature, Zhu and Song [18] derived some sufficient conditions by applying the Lyapunov-Krasovskii functional, Dynkin formula, and Razumikhin technique with a stochastic version as well as the linear matrix inequalities (LMIs) technique. In [19], some new delay-dependent conditions are derived applying the Lyapunov stability theory, Dynkins formula, and linear matrix inequality technique. And the method, linear matrix inequalities technique, is also used in the paper [20]. So far, plenty of sufficient conditions have been deduced to determine the stability of coupled systems and more relevant researches can be seen in $[21,22]$ and the references therein.

However, it is complicated to straightly construct an appropriate Lyapunov function for a specific coupled system, for the reason that the stability of a system depends on not only the nature of the vertex system, but also the network topology. Considering that CSNMRs can be described in a digraph, in which each vertex represents an individual system called vertex system and the directed arcs stand for the interconnections and interactions among vertex systems, 
a novel method based on graph theory, which is used to construct the Lyapunov function, is proposed. The pioneering work based on graph theory to consider the global stability problem for coupled systems on networks has been done by Li et al. $[23,24]$. From then on, plenty of researchers devoted themselves to this method for coupled systems [25-30]. We also have made great efforts in this aspect and achieved some results [31-34].

To the best of the authors' knowledge, the discussion about the exponential stability for CSNMRs is not deep enough, and much room remains to be explored. According to our survey, this method has not been applied to the systems with distributed time delay or reaction-diffusion terms. With few conclusions about the stability of CSNMRs obtained from the new method, we start the present research.

Compared with the previous results on the analysis of exponential stability, the main contribution of this paper is threefold. Firstly, distributed time delay and reaction diffusion are taken into account in the model. Secondly, a graph-theoretic approach is employed to get different kinds of sufficient stability criteria. Thirdly, some conditions that keep from finding a global Lyapunov function directly for CSNMRs are developed, through effective utilization of topological structure and coefficients of CSNMRs.

The remainder of this paper is organized as follows: in Section 2, problem statement and preliminaries are presented; in Section 3, with some theorems and corollaries demonstrated, conditions based on graph theory for the stability of CSNMRs are developed. Ultimately, a numerical simulation is given in Section 4 to show the effectiveness and feasibility of our theoretical results.

\section{Modeling and Preliminaries}

In this section, we will give some useful notations, preliminaries about graph theory, and model descriptions.

2.1. Notations. For convenience, we use the following notations. Write $\mathbb{R}, \mathbb{R}^{n}$ for the set of real numbers and $n$ dimensional Euclidean space. Denote $\mathbb{R}^{+}=[0,+\infty), \mathbb{L}=$ $\{1,2, \ldots, n\}$ and $\mathscr{C}^{1,1}\left(\mathbb{R}^{n} \times \mathbb{R}^{+} ; \mathbb{R}^{+}\right)$for the family of all nonnegative functions $V(x, t)$ on $\mathbb{R}^{n} \times \mathbb{R}^{+}$that are continuously differentiable once in $x$ and once in $t$. Let $\Omega$ be an open bounded domain in $\mathbb{R}^{l}$, which has smooth boundary $\partial \Omega$, and $\operatorname{mes}(\Omega)>0$ denotes the measure of $\Omega$. Denote by $\mathscr{C}^{*} \triangleq \mathscr{C}\left(\left[-\tau_{0}, 0\right] \times \Omega, \mathbb{R}^{n}\right)$ the Banach space of continuous functions mapping $\left[-\tau_{0}, 0\right] \times \Omega$ into $\mathbb{R}^{n}$ with the topology of uniform converge. Set $u(x)=\left(u_{1}(x), u_{2}(x), \ldots, u_{n}(x)\right)^{T}$ and $L^{2}(\Omega)$ to be the space of real functions on $\Omega$ which are $L^{2}$ for the Lebesgue measure. It is a Banach space with the norm

$$
|u(x)|_{2}=\sqrt{\sum_{i=1}^{n}\left|u_{i}(x)\right|_{2}^{2}}
$$

where $\left|u_{i}(x)\right|_{2}=\left(\int_{\Omega}\left|u_{i}(x)\right|^{2} d x\right)^{1 / 2}$. For any $\phi(s, x) \in \mathscr{C}^{*}$, define

$$
|\phi|_{2}=\sqrt{\sum_{i=1}^{n}\left|\phi_{i}\right|_{2}^{2}}
$$

where $\phi(s, x)=\left(\phi_{1}(s, x), \phi_{2}(s, x), \ldots, \phi_{n}(s, x)\right)^{T},\left|\phi_{i}\right|_{2}=$ $\left(\int_{\Omega}\left|\phi_{i}(x)\right|_{\tau_{0}}^{2} d x\right)^{1 / 2}$, and $\left|\phi_{i}(x)\right|_{\tau_{0}}=\sup _{-\tau_{0} \leq s \leq 0}\left|\phi_{i}(s, x)\right|$.

2.2. Basic Concepts on Graph Theory. The following basic concepts on graph theory from [23] will be reviewed. A digraph $\mathscr{G}=(U, E)$ contains a set $U=\{1,2, \ldots, n\}$ of vertices and a set $E$ of $\operatorname{arcs}(i, j)$ leading from initial vertex $i$ to terminal vertex $j$. A subgraph $\mathscr{L}$ of $\mathscr{G}$ is said to be spanning if $\mathscr{L}$ and $\mathscr{G}$ have the same vertex set. A digraph $\mathscr{G}$ is weighted if each $\operatorname{arc}(j, i)$ is assigned a positive weight $h_{i j}$, where $h_{i j}>0$ if and only if there exists an arc from vertex $j$ to vertex $i$ in $\mathscr{G}$. The weight $W(\mathscr{G})$ of $\mathscr{G}$ is the product of the weights on all its arcs. A directed path $\mathscr{P}$ in $\mathscr{G}$ is a subgraph with distinct vertices $\left\{i_{1}, i_{2}, \ldots, i_{s}\right\}$, such that its set of $\operatorname{arcs}$ is $\left\{\left(i_{k}, i_{k+1}\right)\right.$ : $k=1,2, \ldots, s-1\}$. We call $\mathscr{C}$ a directed cycle if $i_{s}=i_{1}$. A connected subgraph $\mathscr{T}$ is a tree if it contains no cycles. A tree $\mathscr{T}$ is rooted at vertex $i$, called the root, if $i$ is not a terminal vertex of any arcs, and each of the remaining vertices is a terminal vertex of exactly one arc. A digraph $\mathscr{G}$ is strongly connected if there exists a directed path from one to the other for any pair of distinct vertices. Given a weighted digraph $\mathscr{G}$ with $n$ vertices, define the weight matrix $A=\left(h_{i j}\right)_{n \times n}$ whose entry $h_{i j}$ equals the weight of $\operatorname{arc}(j, i)$ if it exists, and 0 otherwise. Denote the digraph with weight matrix $A$ as $(\mathscr{G}, A)$. A weighted digraph $(\mathscr{G}, A)$ is said to be balanced if $W(\mathscr{C})=W(-\mathscr{C})$ for all directed cycles $\mathscr{C}$. Here, $-\mathscr{C}$ denotes the reverse of $\mathscr{C}$ and is constructed by reversing the direction of all $\operatorname{arcs}$ in $\mathscr{C}$. For a unicyclic graph $\mathbb{Q}$ with cycle $\mathscr{C}_{\mathscr{Q}}$, let $\widetilde{Q}$ be the unicyclic graph obtained by replacing $\mathscr{C}_{Q}$ with $-\mathscr{C}_{Q}$. Suppose that $(\mathscr{G}, A)$ is balanced; then $W(\widetilde{Q})=W(\widetilde{Q})$. The Laplacian matrix of $(\mathscr{G}, A)$ is defined as

$$
L=\left(\begin{array}{cccc}
\sum_{k \neq 1} a_{1 k} & -a_{12} & \cdots & -a_{1 n} \\
-a_{21} & \sum_{k \neq 2} a_{2 k} & \cdots & -a_{2 n} \\
\vdots & \vdots & \ddots & \vdots \\
-a_{n 1} & -a_{n 2} & \cdots & \sum_{k \neq n} a_{n k}
\end{array}\right) .
$$

Here, we show a result in $\mathrm{Li}$ et al. [23], which will be used in Section 3.

Lemma 1 (see [23]; assume $n \geq 2$ ). Then the following identity holds:

$$
\sum_{i, j=1}^{n} C_{i} h_{i j} F_{i j}\left(x_{i}, x_{j}\right)=\sum_{\mathscr{Q} \in \mathbb{Q}} w(\mathcal{Q}) \sum_{(s, r) \in E\left(C_{Q}\right)} F_{r s}\left(x_{r}, x_{s}\right) .
$$

Here, $C_{i}$ denotes the cofactor of the ith diagonal element of Laplacian matrix of $(\mathscr{G}, A)$. And $F_{i j}\left(x_{i}, x_{j}\right), i, j \in \mathbb{L}$, are arbitrary functions; $\mathbb{Q}$ is the set of all spanning unicyclic graphs 
of $(\mathscr{G}, A), w(Q) \geq 0$ is the weight of $Q$, and $C_{\mathscr{Q}}$ denotes the directed cycle of $Q$. In particular, if $(\mathscr{G}, A)$ is strongly connected, then $C_{i}>0$ for $i \in \mathbb{L}$.

2.3. Model Descriptions. It is well known that CSNMRs have caught many researchers' attention. In this paper, exponential stability for delayed coupled networks is studied as stated in Section 1.

Given a network represented by digraph $\mathscr{G}$ with $n$ vertices, $n \geq 2$, we can get the following system [35]:

$$
\begin{aligned}
\frac{\partial}{\partial t} \tilde{y}_{i}(t, x)= & \sum_{k=1}^{l} \frac{\partial}{\partial x_{k}}\left(D_{i k} \frac{\partial \tilde{y}_{i}(t, x)}{\partial x_{k}}\right)-c_{i} \tilde{y}_{i}(t, x) \\
& +\sum_{j=1}^{n} a_{i j} \tilde{f}_{j}\left(\tilde{y}_{j}(t, x)\right)+\sum_{j=1}^{n} b_{i j} \tilde{g}_{j}\left(\tilde{y}_{j}(t-\tau(t), x)\right) \\
& +\sum_{j=1}^{n} k_{i j} \int_{t-\tau_{1}(t)}^{t} \tilde{h}_{j}\left(\tilde{y}_{j}(s, x)\right) d s+S_{i},
\end{aligned}
$$

where $c_{i}>0$ is a constant, $i \in \mathbb{L}, x=\left(x_{1}, x_{2}, \ldots, x_{l}\right)^{T} \in$ $\Omega \subset \mathbb{R}^{l}$, and $\Omega=\left\{x=\left(x_{1}, x_{2}, \ldots, x_{l}\right)^{T}|| x_{k} \mid \leq m_{k}, k=\right.$ $1,2, \ldots, l\}$ is a bound compact set with smooth boundary $\partial \Omega$, and $\operatorname{mes}(\Omega)>0$ in space $\mathbb{R}^{l} \cdot y_{i}(t, x)$ represents the state of the $i$ th vertex at time $t$ and in space $x . D_{i k} \geq 0$ refers to the transmission diffusion operator along the $i$ th vertex. $a_{i j}, b_{i j}$, and $k_{i j}$ are, respectively, the connection strength, the timevarying delay connection weight, and the distributed timevarying delay connection weight of the $j$ th vertex to the $i$ th vertex. $0<\tau(t) \leq \tau_{0}$ and $0<\tau_{1}(t) \leq \widetilde{\tau}_{1}$ are the time-varying delay and the distributed time-varying delay, respectively.

In order to complete the proof of this paper, we suppose that the system (5) has a trivial solution $\left(y_{1}^{*}, y_{2}^{*}, \ldots, y_{n}^{*}\right)^{T}$. By making a transformation $y_{i}(t, x)=\tilde{y}_{i}(t, x)-y_{i}^{*}$, system $(5)$ can be rewritten as

$$
\begin{aligned}
\frac{\partial}{\partial t} y_{i}(t, x)= & \sum_{k=1}^{l} \frac{\partial}{\partial x_{k}}\left(D_{i k} \frac{\partial y_{i}(t, x)}{\partial x_{k}}\right)-c_{i} y_{i}(t, x) \\
& +\sum_{j=1}^{n} a_{i j} f_{j}\left(y_{j}(t, x)\right)+\sum_{j=1}^{n} b_{i j} g_{j}\left(y_{j}(t-\tau(t), x)\right) \\
& +\sum_{j=1}^{n} k_{i j} \int_{t-\tau_{1}(t)}^{t} h_{j}\left(y_{j}(s, x)\right) d s
\end{aligned}
$$

where $f_{j}\left(y_{j}(\cdot, x)\right)=\widetilde{f}_{j}\left(y_{j}(\cdot, x)\right)-\widetilde{f}_{j}\left(y_{j}^{*}\right), g_{j}\left(y_{j}(\cdot, x)\right)=$ $\widetilde{g}_{j}\left(y_{j}(\cdot, x)\right)-\widetilde{g}_{j}\left(y_{j}^{*}\right)$, and $h_{j}\left(y_{j}(\cdot, x)\right)=\widetilde{h}_{j}\left(y_{j}(\cdot, x)\right)-\widetilde{h}_{j}\left(y_{j}^{*}\right)$.

The boundary conditions of system (6) associated are given by

$$
\left.y_{i}(t, x)\right|_{\partial \Omega}=0, \quad(t, x) \in\left[-\tau_{2},+\infty\right) \times \partial \Omega,
$$

as well as the initial value of system (6):

$$
y_{i}(s, x)=\phi_{i}(s, x), \quad(s, x) \in\left[-\tau_{2}, 0\right] \times \Omega,
$$

where $i \in \mathbb{L}, \tau_{2}=\max \left\{\tau_{0}, \widetilde{\tau}_{1}\right\}$, and $\phi(s, x)=$ $\left(\phi_{1}(s, x), \phi_{2}(s, x), \ldots, \phi_{n}(s, x)\right)^{T}$ is bounded and continuous on $\left[-\tau_{2}, 0\right] \times \Omega$.

Throughout this paper, the following definition, assumptions, and lemmas are needed to derive our main results.

(A1) The functions $\tilde{f}_{j}$ and $\tilde{g}_{j}$ satisfy

$$
\begin{aligned}
& \left|\tilde{f}_{j}\left(u_{j}\right)-\tilde{f}_{j}\left(v_{j}\right)\right| \leqslant P_{j}\left|u_{j}-v_{j}\right|, \\
& \left|\tilde{g}_{j}\left(u_{j}\right)-\tilde{g}_{j}\left(v_{j}\right)\right| \leqslant Q_{j}\left|u_{j}-v_{j}\right|, \\
& \left|\widetilde{h}_{j}\left(u_{j}\right)-\widetilde{h}_{j}\left(v_{j}\right)\right| \leqslant T_{j}\left|u_{j}-v_{j}\right|,
\end{aligned}
$$

for any $u_{j}, v_{j} \in \mathbb{R}, j \in \mathbb{L}$, where $P_{j}, Q_{j}$, and $T_{j}$ are positive constants.

(A2) $\tau(t)$ and $\tau_{1}(t)$, time-varying delays, satisfy $\dot{\tau}(t) \leqslant \gamma<$ 1 and $\dot{\tau}_{1}(t) \leqslant \gamma^{*}<1$ for all $t$, respectively, where $\gamma$ and $\gamma^{*}$ are constants.

Definition 2. The trivial solution to system (6) is said to be exponential stable, if there exist positive constants $L, \beta$, and $p \geq 2$ such that

$$
|y(t, x)|_{2}^{p} \leq L e^{-\beta t}
$$

for $(t, x) \in[0,+\infty) \times \Omega$.

Lemma 3 (see [35]). Let $X$ be a cube $\left|x_{k}\right| \leq m_{k}(k=$ $1,2, \ldots, l)$, and let $\varphi(x)$ be a real-valued function belonging to $\mathscr{C}^{1}(\Omega)$ which vanishes on the boundary $\partial \Omega$ of $\Omega$; that is, $\left.\varphi(x)\right|_{\partial \Omega}=0$. Then

$$
\int_{\Omega} \varphi^{2}(x) d x \leq m_{k}^{2} \int_{\Omega}\left|\frac{\partial \varphi}{\partial x_{k}}\right|^{2} d x .
$$

Lemma 4 (see [36]). For any positive definite matrix $G>0$, scalar $\tau>0$, and vector function $\omega:[0, \tau] \rightarrow \mathbb{R}^{n}$ such that the integrations concerned are well defined, the following inequality holds:

$$
\left[\int_{0}^{\tau} \omega(s) d s\right]^{T} G\left[\int_{0}^{\tau} \omega(s) d s\right] \leq \tau \int_{0}^{\tau} \omega^{T}(s) G \omega(s) d s .
$$

\section{Stability Analysis}

In the study of stability, Lyapunov method plays an important role. Combining Lyapunov method with graph theory, two kinds of sufficient criteria are investigated. One is given in the form of Lyapunov function, while the other is in terms of coefficients of system (6).

3.1. Lyapunov-Type Theorem. In order to propose a Lyapunov function for system (6), we first give a definition about vertexLyapunov functions on the basis of [37].

Definition 5. Functions $V_{i}\left(y_{i}, t\right) \in \mathscr{C}^{1,1}\left(\mathbb{R} \times \mathbb{R}^{+} ; \mathbb{R}^{+}\right)$for $i \in \mathbb{L}$, as

$$
V_{i}\left(y_{i}, t\right)=e^{\beta t} V_{i}^{(1)}\left(y_{i}, t\right)+V_{i}^{(2)}(t),
$$


in which $\beta>0, V_{i}^{(2)}(t) \geq 0$, are called vertex-Lyapunov functions for system (6) if the following hold.

(H1) There are positive constants $p \geq 2, \alpha_{i}, \beta_{i}$, where $i \in \mathbb{L}$, functions $F_{i j}\left(y_{i}, y_{j}\right)$, and matrix $A=\left(h_{i j}\right)_{n \times n}, h_{i j} \geq 0$ satisfying

$$
\begin{aligned}
\alpha_{i}\left|y_{i}\right|^{p} \leq & V_{i}^{(1)}\left(y_{i}, t\right) \leq \beta_{i}\left|y_{i}\right|^{p}, \\
\frac{\partial}{\partial t} V_{i}\left(y_{i}(t, x), t\right)= & \frac{\partial}{\partial t} V_{i}\left(y_{i}, t\right) \\
& +\frac{\partial}{\partial y_{i}} V_{i}\left(y_{i}, t\right) \frac{\partial}{\partial t} y_{i}(t, x) \\
\leq & e^{\beta t} \sum_{j=1}^{n} h_{i j} F_{i j}\left(y_{i}, y_{j}\right) .
\end{aligned}
$$

(H2) Along each directed cycle $\mathscr{C}$ of weighted digraph $(\mathscr{G}, A)$, there is

$$
\sum_{(s, r) \in E(\mathscr{C})} F_{r s}\left(y_{r}, y_{s}\right) \leq 0
$$

for all $y_{r} \in \mathbb{R}, y_{s} \in \mathbb{R}$.

Theorem 6. Let $(\mathscr{G}, A)$ be strongly connected. If system (6) admits vertex-Lyapunov functions $V_{i}$, then the trivial solution of system (6) is exponentially stable.

Proof. Let $V(y, t)=\sum_{i=1}^{n} C_{i} V_{i}\left(y_{i}, t\right)$, where $C_{i}$ is defined as Lemma 1. Denote

$$
\alpha=\left(\min _{i \in \mathbb{L}}\left\{C_{i} \alpha_{i}\right\}\right)^{2 / p}
$$

Note that weighted digraph $(\mathscr{G}, A)$ is strongly connected, which implies that $C_{i}>0$ for any $i \in \mathbb{L}$. So $\alpha$ is a positive number. Then, it follows readily from conditions (13) and (14) that

$$
\begin{aligned}
& V(y, t) \\
& \quad=\sum_{i=1}^{n} C_{i} e^{\beta t} V_{i}^{(1)}\left(y_{i}, t\right)+\sum_{i=1}^{n} C_{i} V_{i}^{(2)}(t) \\
& \geq e^{\beta t} \sum_{i=1}^{n} C_{i} \alpha_{i}\left|y_{i}\right|^{p}+\sum_{i=1}^{n} C_{i} V_{i}^{(2)}(t) \\
& \quad \geq e^{\beta t} \sum_{i=1}^{n} C_{i} \alpha_{i}\left|y_{i}\right|^{p} .
\end{aligned}
$$

Meanwhile, taking the partial derivative of $V(y(t, x), t)$, via inequalities (15) and (16), $w(Q) \geq 0$, and Lemma 1 , we deduce

$$
\begin{aligned}
\int_{\Omega} \frac{\partial}{\partial t} & V(y, t) d x \\
& =\int_{\Omega} \sum_{i=1}^{n} C_{i} \frac{\partial}{\partial t} V_{i}\left(y_{i}, t\right) d x \\
& \leq e^{\beta t} \int_{\Omega} \sum_{i=1}^{n} \sum_{j=1}^{n} C_{i} h_{i j} F_{i j}\left(y_{i}, y_{j}\right) d x \\
& =e^{\beta t} \int_{\Omega} \sum_{Q \in \mathbb{Q}} w(Q) \sum_{(s, r) \in E\left(C_{\Theta}\right)} F_{r s}\left(y_{r}, y_{s}\right) d x \leq 0 .
\end{aligned}
$$

Thereby,

$$
\begin{aligned}
\int_{\Omega} V(y(t, x), t) d x \\
\quad \leq \int_{\Omega} V(y(0, x), 0) d x \\
\quad=\int_{\Omega} \sum_{i=1}^{n} C_{i}\left(V_{i}^{(1)}\left(y_{i}(0), 0\right)+V_{i}^{(2)}(0)\right) d x .
\end{aligned}
$$

Combining Hölder inequality and (18), we successively find that

$$
\begin{aligned}
C_{0} \alpha e^{\beta t}|y(t, x)|_{2}^{p} & \leq \int_{\Omega} e^{\beta t} \sum_{i=1}^{n} C_{i} \alpha_{i}\left|y_{i}\right|^{p} d x \\
& \leq \int_{\Omega} V(y(t, x), t) d x \\
& \leq \int_{\Omega} \sum_{i=1}^{n} C_{i}\left(V_{i}^{(1)}\left(y_{i}(0), 0\right)+V_{i}^{(2)}(0)\right) d x,
\end{aligned}
$$

where $C_{0}$ is a positive constant. In the sequel, define

$$
L=C_{0}^{-1} \alpha^{-1} \int_{\Omega} \sum_{i=1}^{n} C_{i}\left(V_{i}^{(1)}\left(y_{i}(0), 0\right)+V_{i}^{(2)}(0)\right) d x,
$$

where $L>0$, evidently. Hence, after rearrangement, we obtain

$$
|y(t, x)|_{2}^{p} \leq L e^{-\beta t}
$$

for all $t \geq 0$. This means that the trivial solution of system (6) is exponentially stable. This concludes the whole proof.

Remark 7. Recently, attention was paid to stability analysis for coupled systems increasingly, and many methods were proposed. Gan and $\mathrm{Xu}$ [8] discussed the local stability by analyzing the corresponding characteristic equations, and more scholars [5, 20-22] used linear matrix inequality approach to construct the Lyapunov function of a system. As is well-known to us, it is a challenging task to construct an appropriate Lyapunov function. In this paper, a new method 
based on graph-theoretic approach, which is first applied to the system with distributed time delay or reaction-diffusion terms, is presented by making use of the Lyapunov function $V_{i}$ of each vertex system. Through effective utilization of topological structure, the method is practicable avoiding finding a Lyapunov function directly for system (6). The validity of the technique is shown in Theorem 10 in Section 3.

However, the conditions in Theorem 6 can be simplified. Now, some simply and easy-verified conditions are discussed. Note that if $(\mathscr{G}, A)$ is balanced, then

$$
\begin{aligned}
& \sum_{i, j=1}^{n} C_{i} h_{i j} F_{i j}\left(x_{i}, x_{j}\right) \\
& \quad=\frac{1}{2} \sum_{\mathbb{Q} \in \mathbb{Q}} w(Q) \sum_{(s, r) \in E\left(\mathscr{C}_{Q}\right)}\left[F_{s r}\left(x_{s}, x_{r}\right)+F_{r s}\left(x_{r}, x_{s}\right)\right] .
\end{aligned}
$$

In this case, condition (H2) can be replaced by

$$
\sum_{(s, r) \in E\left(\mathscr{C}_{Q}\right)}\left[F_{s r}\left(x_{s}, x_{r}\right)+F_{r s}\left(x_{r}, x_{s}\right)\right] \leq 0 .
$$

Consequently, an easier stability criterion is derived below.

Corollary 8. Suppose that $(\mathscr{G}, A)$ is balanced. Then the conclusion of Theorem 6 holds if inequality (16) is replaced by inequality (25).

Furthermore, in view that if for every $s, r \in \mathbb{L}$ there exist functions $P_{s}\left(x_{s}\right)$ and $P_{r}\left(x_{r}\right)$, such that

$$
F_{s r}\left(x_{s}, x_{r}\right) \leq P_{s}\left(x_{s}\right)-P_{r}\left(x_{r}\right)
$$

then inequality (16) follows naturally. Thus, we obtain one more corollary below.

Corollary 9. The conclusion of Theorem 6 holds if inequality (16) is replaced by inequality (26).

Since the previous results are based on vertex-Lyapunov functions for system (6), the exponential stability criteria are not very convenient to be verified for given systems. We now establish some sufficient conditions for exponential stability by using coefficients of system (6) and this method is proved to be very useful in practice.

3.2. Coefficients-Type Theorem. In the following, another sufficient exponential stability criterion is established in the form of coefficients of system (6). For simplicity, we set $\delta_{i}=$ $\sum_{j=1}^{n} b_{i j}^{2}+1, \lambda_{i}=(1-\gamma)\left(\sum_{j=1}^{n} k_{i j}^{2}+1\right)$ and $\sigma_{i}=\sum_{j=1}^{n}\left(\left|a_{i j}\right|+\right.$ $\left.\left|a_{i j}\right| P_{i j}^{2}+Q_{j}^{2}+\widetilde{\tau}_{1}^{2} T_{j}^{2}\right)-2 c_{i}$, with $i \in \mathbb{L}$.

Theorem 10. Let $(\mathscr{G}, A)$ be strongly connected. If $\left(1-\gamma^{*}\right) \delta_{i}+$ $\lambda_{i}+(1-\gamma)\left(1-\gamma^{*}\right) \sigma_{i} \leq 0$, then the trivial solution of system (6) is exponentially stable under hypotheses (A1) and (A2).
Proof. Let

$$
\begin{gathered}
V_{i}^{(1)}\left(y_{i}, t\right)=y_{i}^{2}, \\
V_{i}^{(2)}(t)=\sum_{j=1}^{n} \int_{t-\tau(t)}^{t} e^{2 \mu s}\left[g_{j}\left(y_{j}(s, x)\right)\right]^{2} d s \\
+\int_{t-\tau(t)}^{t} \frac{e^{2 \mu \tau_{0}}}{1-\gamma} e^{2 \mu s} y_{i}^{2}(s, x) d s \\
+\widetilde{\tau}_{1} \sum_{j=1}^{n} \int_{-\tau_{1}(t)}^{0} \int_{t+s}^{t} e^{2 \mu \theta}\left[h_{j}\left(y_{j}(\theta, x)\right)\right]^{2} d \theta d s \\
+\frac{e^{2 \mu \tau_{1}}}{1-\gamma^{*}} \int_{t-\tau_{1}(t)}^{t} e^{2 \mu s} y_{i}^{2}(s, x) d s,
\end{gathered}
$$

where $i \in \mathbb{L}$ and $\mu>0$ is an enough small real number properly selected. With $\beta=2 \mu$, it is explicit that $\beta>0$ and $V_{i}^{(2)}(t) \geq 0$. Then, from equality (13), it follows that

$$
\begin{aligned}
V_{i}\left(y_{i}, t\right)= & e^{2 \mu t} y_{i}^{2}+\sum_{j=1}^{n} \int_{t-\tau(t)}^{t} e^{2 \mu s}\left[g_{j}\left(y_{j}(s, x)\right)\right]^{2} d s \\
& +\int_{t-\tau(t)}^{t} \frac{e^{2 \mu \tau_{0}}}{1-\gamma} e^{2 \mu s} y_{i}^{2}(s, x) d s \\
& +\widetilde{\tau}_{1} \sum_{j=1}^{n} \int_{-\tau_{1}(t)}^{0} \int_{t+s}^{t} e^{2 \mu \theta}\left[h_{j}\left(y_{j}(\theta, x)\right)\right]^{2} d \theta d s \\
& +\frac{e^{2 \mu \tau_{1}}}{1-\gamma^{*}} \int_{t-\tau_{1}(t)}^{t} e^{2 \mu s} y_{i}^{2}(s, x) d s .
\end{aligned}
$$

Then obviously,

$$
\begin{aligned}
& \int_{\Omega} \frac{\partial}{\partial t} V_{i}\left(y_{i}(t, x), t\right) d x \\
& =\int_{\Omega}\left[2 \mu e^{2 \mu t} y_{i}^{2}(t, x)\right. \\
& \quad+\sum_{j=1}^{n}\left(e^{2 \mu t}\left[g_{j}\left(y_{j}(t, x)\right)\right]^{2}-(1-\dot{\tau}(t))\right. \\
& \left.\quad \times e^{2 \mu(t-\tau(t))}\left[g_{j}\left(y_{j}(t-\tau(t), x)\right)\right]^{2}\right)+\frac{e^{2 \mu \tau_{0}}}{1-\gamma} \\
& \quad \times\left(e^{2 \mu t} y_{i}^{2}(t, x)-(1-\dot{\tau}(t)) e^{2 \mu(t-\tau(t))} y_{i}^{2}(t-\tau(t), x)\right) \\
& +2 e^{2 \mu t} y_{i}(t, x) \sum_{k=1}^{l} \frac{\partial}{\partial x_{k}}\left(D_{i k} \frac{\partial y_{i}(t, x)}{\partial x_{k}}\right)
\end{aligned}
$$




$$
\begin{gathered}
+2 e^{2 \mu t} y_{i}(t, x)\left(-c_{i} y_{i}(t, x)+\sum_{j=1}^{n} a_{i j} f_{j}\left(y_{j}(t, x)\right)\right. \\
+\sum_{j=1}^{n} b_{i j} g_{j}\left(y_{j}(t-\tau(t), x)\right) \\
\left.+\sum_{j=1}^{n} k_{i j} \int_{t-\tau_{1}(t)}^{t} h_{j}\left(y_{j}(s, x)\right) d s\right) \\
+\sum_{j=1}^{n} \widetilde{\tau}_{1}\left(\tau_{1}(t) e^{2 \mu t}\left[h_{j}\left(y_{j}(t, x)\right)\right]^{2}-\left(1-\dot{\tau}_{1}(t)\right)\right. \\
\left.\times \int_{t-\tau_{1}(t)}^{t} e^{2 \mu s}\left[h_{j}\left(y_{j}(s, x)\right)\right]^{2} d s\right) \\
+\frac{e^{2 \mu \tau_{1}}}{1-\gamma^{*}}\left(e^{2 \mu t} y_{i}^{2}(t, x)-\left(1-\dot{\tau}_{1}(t)\right) e^{2 \mu\left(t-\tau_{1}(t)\right)}\right. \\
\left.\left.\times y_{i}^{2}\left(t-\tau_{1}(t), x\right)\right)\right] d x .
\end{gathered}
$$

$$
\begin{aligned}
& \times\left(-c_{i} y_{i}(t, x)+\sum_{j=1}^{n} a_{i j} f_{j}\left(y_{j}(t, x)\right)\right. \\
& +\sum_{j=1}^{n} b_{i j} g_{j}\left(y_{j}(t-\tau(t), x)\right) \\
& \left.\left.+\sum_{j=1}^{n} k_{i j} \int_{t-\tau_{1}(t)}^{t} h_{j}\left(y_{j}(s, x)\right) d s\right)\right] d x \\
& \left.-2 \sum_{k=1}^{l} \int_{\Omega} \frac{D_{i k}}{m_{k}^{2}} y_{i}^{2}(t, x) d x\right\} .
\end{aligned}
$$

From Lemma 4, it follows that

Using the boundary conditions (7) and Lemma 3, we can get $[17,35]$

$$
\begin{gathered}
\int_{\Omega} 2 e^{2 \mu t} y_{i}(t, x) \sum_{k=1}^{l} \frac{\partial}{\partial x_{k}}\left(D_{i k} \frac{\partial y_{i}(t, x)}{\partial x_{k}}\right) d x \\
\leq-2 e^{2 \mu t} \sum_{k=1}^{l} \int_{\Omega} \frac{D_{i k}}{m_{k}^{2}} y_{i}^{2}(t, x) d x .
\end{gathered}
$$

Proceeding by applying inequality (30) and (A2) to equality (29), we obtain

$$
\begin{gathered}
\int_{\Omega} \frac{\partial}{\partial t} V_{i}\left(y_{i}(t, x), t\right) d x \\
\leq e^{2 \mu t}\left\{\int _ { \Omega } \left[2 \mu y_{i}^{2}(t, x)\right.\right. \\
+\sum_{j=1}^{n}\left(\left[g_{j}\left(y_{j}(t, x)\right)\right]^{2}-(1-\gamma) e^{-2 \mu \tau_{0}}\right. \\
\times\left[g_{j}\left(y_{j}(t-\tau(t), x)\right)\right]^{2} \\
+\widetilde{\tau}_{1}^{2}\left[h_{j}\left(y_{j}(t, x)\right)\right]^{2}-\widetilde{\tau}_{1}\left(1-\gamma^{*}\right) e^{-2 \mu \tau_{1}} \\
\left.\times \int_{t-\tau_{1}(t)}^{t}\left[h_{j}\left(y_{j}(s, x)\right)\right]^{2} d s\right)+\frac{e^{2 \mu \tau_{0}}}{1-\gamma} \\
\times\left(y_{i}^{2}(t, x)-(1-\gamma) e^{-2 \mu \tau_{0}} y_{i}^{2}(t-\tau(t), x)\right) \\
+\frac{e^{2 \mu \tau_{1}}}{1-\gamma^{*}}\left(y_{i}^{2}(t, x)-\left(1-\gamma^{*}\right) e^{-2 \mu \widetilde{\tau}_{1}}\right. \\
\left.\times y_{i}^{2}\left(t-\tau_{1}(t), x\right)\right)+2 y_{i}(t, x)
\end{gathered}
$$$$
-\sum_{j=1}^{n} \widetilde{\tau}_{1}\left(1-\gamma^{*}\right) e^{-2 \mu \widetilde{\tau}_{1}} \int_{t-\tau_{1}(t)}^{t}\left[h_{j}\left(y_{j}(s, x)\right)\right]^{2} d s
$$$$
+2 \sum_{j=1}^{n} k_{i j} y_{i}(t, x) \int_{t-\tau_{1}(t)}^{t} h_{j}\left(y_{j}(s, x)\right) d s
$$$$
\leq-\sum_{j=1}^{n}\left(1-\gamma^{*}\right) e^{-2 \mu \tau_{1}}\left[\int_{t-\tau_{1}(t)}^{t} h_{j}\left(y_{j}(s, x)\right) \mathrm{d} s\right]^{2}
$$$$
+2 \sum_{j=1}^{n} k_{i j} y_{i}(t, x) \int_{t-\tau_{1}(t)}^{t} h_{j}\left(y_{j}(s, x)\right) d s
$$$$
=-\sum_{j=1}^{n}\left[\left(1-\gamma^{*}\right)^{1 / 2} e^{-\mu \widetilde{\tau}_{1}} \int_{t-\tau_{1}(t)}^{t} h_{j}\left(y_{j}(s, x)\right) d s\right.
$$$$
\left.-\left(1-\gamma^{*}\right)^{-1 / 2} e^{\mu \tilde{\tau}_{1}} k_{i j} y_{i}(t, x)\right]^{2}
$$$$
+\frac{e^{2 \mu \tilde{\tau}_{1}}}{1-\gamma^{*}} \sum_{j=1}^{n} k_{i j}^{2} y_{i}^{2}(t, x)
$$$$
\leq \frac{e^{2 \mu \widetilde{\tau}_{1}}}{1-\gamma^{*}} \sum_{j=1}^{n} k_{i j}^{2} y_{i}^{2}(t, x),
$$

$$
2 \sum_{j=1}^{n} a_{i j} y_{i}(t, x) f_{j}\left(y_{j}(t, x)\right)
$$$$
\leq \sum_{j=1}^{n}\left|a_{i j}\right|\left[y_{i}^{2}(t, x)+\left(f_{j}\left(y_{j}(t, x)\right)\right)^{2}\right] \text {, }
$$ 


$$
\begin{aligned}
& -\sum_{j=1}^{n}(1-\gamma) e^{-2 \mu \tau_{0}}\left[g_{j}\left(y_{j}(t-\tau(t), x)\right)\right]^{2} \\
& +2 \sum_{j=1}^{n} b_{i j} y_{i}(t, x) g_{j}\left(y_{j}(t-\tau(t), x)\right) \\
& =-\sum_{j=1}^{n}\left[(1-\gamma)^{-1 / 2} e^{-\mu \tau_{0}} g_{j}\left(y_{j}(t-\tau(t), x)\right)\right. \\
& +\frac{e^{2 \mu \tau_{0}}}{1-\gamma} \sum_{j=1}^{n} b_{i j}^{2} y_{i}^{2}(t, x) \\
& \leq \frac{e^{2 \mu \tau_{0}}}{1-\gamma} \sum_{j=1}^{n} b_{i j}^{2} y_{i}^{2}(t, x) .
\end{aligned}
$$

Proceeding again by inequalities (31)-(33) and (A1), given $h_{i j}=\left|a_{i j}\right| P_{j}^{2}+Q_{j}^{2}+\widetilde{\tau}_{1}^{2} T_{j}^{2}, F_{i j}\left(y_{i}, y_{j}\right)=y_{j}^{2}-y_{i}^{2}$, we find

$$
\begin{aligned}
& \int_{\Omega} \frac{\partial}{\partial t} V_{i}\left(y_{i}(t, x), t\right) d x \\
& \leq e^{2 \mu t} \int_{\Omega}\left\{\left[2 \mu+\frac{e^{2 \mu \tau_{0}}}{1-\gamma}\left(\sum_{j=1}^{n} b_{i j}^{2}+1\right)+\frac{e^{2 \mu \tau_{1}}}{1-\gamma^{*}}+\sum_{j=1}^{n}\left|a_{i j}\right|\right.\right. \\
& \left.+\frac{e^{2 \mu \widetilde{\tau}_{1}}}{1-\gamma^{*}} \sum_{j=1}^{n} k_{i j}^{2}-2 c_{i}\right] y_{i}^{2}(t, x) \\
& \left.+\sum_{j=1}^{n}\left(\left|a_{i j}\right| P_{j}^{2}+Q_{j}^{2}+\widetilde{\tau}_{1}^{2} T_{j}^{2}\right) y_{j}^{2}(t, x)\right\} d x \\
& =e^{2 \mu t} \int_{\Omega}\left\{\left[2 \mu+\frac{e^{2 \mu \tau_{0}}}{1-\gamma}\left(\sum_{j=1}^{n} b_{i j}^{2}+1\right)\right.\right. \\
& +\frac{e^{2 \mu \tilde{\tau}_{1}}}{1-\gamma^{*}}\left(\sum_{j=1}^{n} k_{i j}^{2}+1\right) \\
& \left.-2 c_{i}+\sum_{j=1}^{n}\left|a_{i j}\right|+\sum_{j=1}^{n} h_{i j}\right] y_{i}^{2}(t, x) \\
& \left.+\sum_{j=1}^{n} h_{i j} F_{i j}\left(y_{i}, y_{j}\right)\right\} d x \\
& =e^{2 \mu t} \int_{\Omega}\left\{\left[2 \mu+\frac{e^{2 \mu \tau_{0}}}{1-\gamma}\left(\delta_{i}+\frac{e^{2 \mu \tilde{\tau}_{1}}}{1-\gamma^{*}} \lambda_{i}\right)+\sigma_{i}\right]\right. \\
& \left.\times y_{i}^{2}(t, x)+\sum_{j=1}^{n} h_{i j} F_{i j}\left(y_{i}, y_{j}\right)\right\} d x
\end{aligned}
$$

With $\left(1-\gamma^{*}\right) \delta_{i}+\lambda_{i}+(1-\gamma)\left(1-\gamma^{*}\right) \sigma_{i}<0$, it is obvious that $2 \mu+\left(e^{2 \mu \tau_{0}} /(1-\gamma)\right)\left(\delta_{i}+\left(e^{2 \mu \tau_{1}} /\left(1-\gamma^{*}\right)\right) \lambda_{i}\right)+\sigma_{i} \leq 0$, when $\mu>0$ is an enough small real number. As a consequence, inequality (34) can be changed into

$$
\int_{\Omega} \frac{\partial}{\partial t} V_{i}\left(t, y_{i}(t, x)\right) d x \leq \int_{\Omega} e^{2 \mu t} \sum_{j=1}^{n} h_{i j} F_{i j}\left(y_{i}, y_{j}\right) d x
$$

On the other hand, along each directed cycle $\mathscr{C}$ of weighted digraph $\left(\mathscr{G}, A_{n \times n}\right)$, there is $\sum_{(s, r) \in E(\mathscr{C})} F_{r s}\left(y_{r}-y_{s}\right)=$ $\sum_{(s, r) \in E(\mathscr{C})}\left(y_{s}^{2}-y_{r}^{2}\right)=0$. Therefore, from Theorem 6 , one can conclude that the trivial solution of system (6) is exponentially stable.

\section{Numerical Test}

In this section, we will give an example, showing the effectiveness and the correctness of our results. Consider the following system:

$$
\begin{aligned}
\frac{\partial y_{i}(t, x)}{\partial t}= & D_{i} \frac{\partial^{2} y_{i}(t, x)}{\partial x^{2}}-c_{i} y_{i}(t, x) \\
& +\sum_{j=1}^{3} a_{i j} f_{j}\left(y_{j}(t, x)\right)+\sum_{j=1}^{3} b_{i j} g_{j}\left(y_{j}(t-\tau, x)\right) \\
& +\sum_{j=1}^{3} k_{i j} \int_{t-\tau_{1}(t)}^{t} h_{j}\left(y_{j}(s, x)\right) d s,
\end{aligned}
$$

where $i=1,2,3$, and $f_{j}\left(y_{j}\right)=g_{j}\left(y_{j}\right)=h_{j}\left(y_{j}\right)=\tanh \left(y_{j}\right)$. The parameters of system (36) assumed that $c_{1}=c_{2}=c_{3}=3$, $a_{11}=1.8, a_{12}=1.3, a_{13}=-1.5, a_{21}=-1.5, a_{22}=2, a_{23}=1.1$, $a_{31}=0.6, a_{32}=-2.4, a_{33}=-0.47, b_{11}=-1.5, b_{12}=-0.5$, $b_{13}=-0.1, b_{21}=-0.5, b_{22}=-1, b_{23}=-0.4, b_{31}=-0.37$, $b_{32}=-0.09, b_{33}=-0.1, k_{11}=0.6, k_{12}=0.2, k_{13}=0.3$, $k_{21}=-1, k_{22}=-0.1, k_{23}=-0.05, k_{31}=0.2, k_{32}=0.04, k_{33}=$ $0.01, \tau_{0}=1, \widetilde{\tau}_{1}=1, D 1=0.5, D 2=0.2, D 3=0.1$. We can easily check that the conditions in Theorem 10 are satisfied. Further, we choose the following initial conditions associated with system (36):

$$
\begin{aligned}
& y_{1}(s, x)=9 \cos (2 s) \sin (x \pi)^{2}, \\
& y_{2}(s, x)=-5 \cos (2 s) \sin (x \pi)^{2}, \\
& y_{3}(s, x)=-6.5 \cos (s \pi) \sin (x \pi)^{2},
\end{aligned}
$$

where $(s, x) \in[-1.3,0] \times \Omega$. The solution of system (36) is shown in Figures 1, 2, and 3, with boundary conditions (7) and initial conditions (37). In fact, we can clearly see from Figures 1-3 that the trivial solution of system (36) is exponentially stable. The numerical simulation results verify the effectiveness and feasibility of the proposed results. 


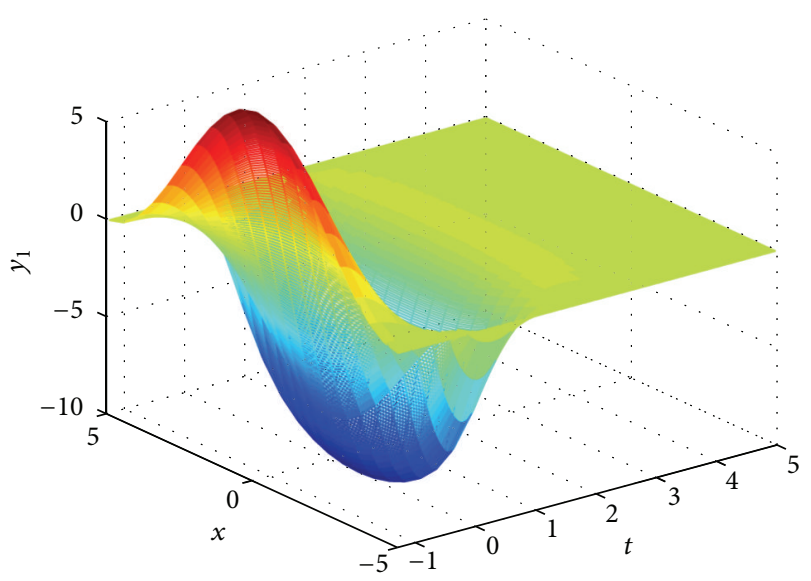

FIgURE 1: The dynamical behavior of the subsystem $y_{1}$ of system (36).

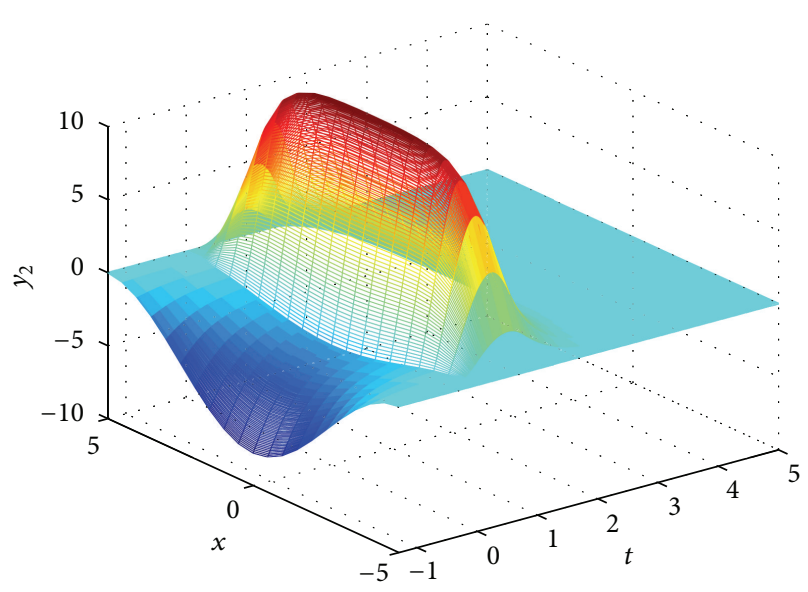

FIGURE 2: The dynamical behavior of the subsystem $y_{2}$ of system (36).

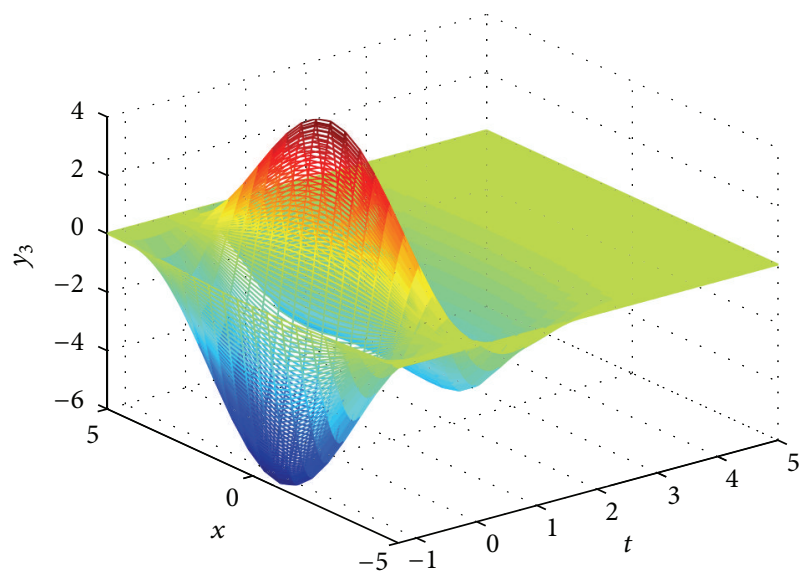

Figure 3: The dynamical behavior of the subsystem $y_{3}$ of system (36).

\section{Discussions}

In this paper, we have investigated the stability of CSNMRs. By applying some results in graph theory and Lyapunov method, we have derived two different types of novel exponential stability criteria. One is given in the form of Lyapunov functions and network topology, while the other is given in the form of coefficients of systems. Compared with the previous stability method, graph-theoretic approach in this paper is new and efficient. Furthermore, an illustrative example is given to validate the approach.

\section{Conflict of Interests}

The authors declare that there is no conflict of interests regarding the publication of this paper.

\section{Acknowledgments}

This work was supported by the NNSF of China (nos. 11301112, 11171081, and 11171056), China Postdoctoral Science Foundation funded project (no. 2013M541352), and the NNSF of Shandong Province (no. ZR2013AQ003).

\section{References}

[1] S. H. Strogatz, "Exploring complex networks," Nature, vol. 410, no. 6825 , pp. 268-276, 2001.

[2] S. Boccaletti, V. Latora, Y. Moreno, M. Chavez, and D.-U. Hwang, "Complex networks: structure and dynamics," Physics Reports, vol. 424, no. 4-5, pp. 175-308, 2006.

[3] W. He and J. Cao, "Exponential synchronization of hybrid coupled networks with delayed coupling," IEEE Transactions on Neural Networks, vol. 21, no. 4, pp. 571-583, 2010.

[4] W. Yu, J. Cao, and W. Lu, "Synchronization control of switched linearly coupled neural networks with delay," Neurocomputing, vol. 73, no. 4-6, pp. 858-866, 2010.

[5] Q. Zhu and J. Cao, "Stability analysis for stochastic neural networks of neutral type with both Markovian jump parameters and mixed time delays," Neurocomputing, vol. 73, no. 13-15, pp. 2671-2680, 2010.

[6] Q. Meng and H. Jiang, "Robust stochastic stability analysis of Markovian switching genetic regulatory networks with discrete and distributed delays," Neurocomputing, vol. 74, no. 1-3, pp. 362-368, 2010.

[7] Q. Zhu, C. Huang, and X. Yang, "Exponential stability for stochastic jumping BAM neural networks with time-varying and distributed delays," Nonlinear Analysis: Hybrid Systems, vol. 5, no. 1, pp. 52-77, 2011.

[8] Q. Gan and R. Xu, "Stability and Hopf bifurcation of a delayed reaction-diffusion neural network," Mathematical Methods in the Applied Sciences, vol. 34, no. 12, pp. 1450-1459, 2011.

[9] Y. Liu, Z. Wang, and X. Liu, "Stability analysis for a class of neutral-type neural networks with Markovian jumping parameters and mode-dependent mixed delays," Neurocomputing, vol. 94, pp. 46-53, 2012.

[10] C. Hu, H. Jiang, and Z. Teng, "Globally exponential stability for delayed neural networks under impulsive control," Neural Processing Letters, vol. 31, no. 2, pp. 105-127, 2010. 
[11] W. Yu, J. Cao, and J. Lü, "Global synchronization of linearly hybrid coupled networks with time-varying delay," SIAM Journal on Applied Dynamical Systems, vol. 7, no. 1, pp. 108-133, 2008.

[12] M. Hua, X. Liu, F. Deng, and J. Fei, "New results on robust exponential stability of uncertain stochastic neural networks with mixed time-varying delays," Neural Processing Letters, vol. 32, no. 3, pp. 219-233, 2010.

[13] Q. Gan, "Exponential synchronization of stochastic CohenGrossberg neural networks with mixed time-varying delays and reaction-diffusion via periodically intermittent control," Neural Networks, vol. 31, pp. 12-21, 2012.

[14] Y. Liu and H. Jiang, "Exponential stability of genetic regulatory networks with mixed delays by periodically intermittent control," Neural Computing and Applications, vol. 21, no. 6, pp. 12631269, 2012.

[15] Q. Gan, "Exponential synchronization of stochastic fuzzy cellular neural networks with reaction-diffusion terms via periodically intermittent control," Neural Processing Letters, vol. 37, pp. 393-410, 2013.

[16] Q. Zhu and J. Cao, "Exponential stability analysis of stochastic reaction-diffusion Cohen-Grossberg neural networks with mixed delays," Neurocomputing, vol. 74, no. 17, pp. 3084-3091, 2011.

[17] F. Yu and H. Jiang, "Global exponential synchronization of fuzzy cellular neural networks with delays and reaction-diffusion terms," Neurocomputing, vol. 74, no. 4, pp. 509-515, 2011.

[18] Q. Zhu and B. Song, "Exponential stability of impulsive nonlinear stochastic differential equations with mixed delays," Nonlinear Analysis: Real World Applications, vol. 12, no. 5, pp. 2851-2860, 2011.

[19] Q. Zhu and J. Cao, "Stability of Markovian jump neural networks with impulse control and time varying delays," Nonlinear Analysis: Real World Applications, vol. 13, no. 5, pp. 2259-2270, 2012.

[20] M. A. Rodrigues and D. Odloak, "New robust stable MPC using linear matrix inequalities," Brazilian Journal of Chemical Engineering, vol. 17, no. 1, pp. 51-69, 2000.

[21] D. Lee, Y. Joo, and M. Tak, "Linear matrix inequality approach to local stability analysis of discrete-time Takagi-Sugeno fuzzy systems," IET Control Theory and Applications, vol. 7, pp. 13091318, 2013.

[22] H. Wang, Q. Song, and C. Duan, "LMI criteria on exponential stability of BAM neural networks with both time-varying delays and general activation functions," Mathematics and Computers in Simulation, vol. 81, no. 4, pp. 837-850, 2010.

[23] M. Y. Li and Z. Shuai, "Global-stability problem for coupled systems of differential equations on networks," Journal of Differential Equations, vol. 248, no. 1, pp. 1-20, 2010.

[24] H. Guo, M. Y. Li, and Z. Shuai, "A graph-theoretic approach to the method of global Lyapunov functions," Proceedings of the American Mathematical Society, vol. 136, no. 8, pp. 2793-2802, 2008.

[25] H. Guo, M. Li, and Z. Shuai, "Global dynamics of a general class of multistage models for infectious diseases," SIAM Journal on Applied Mathematics, vol. 72, pp. 261-279, 2012.

[26] M. Y. Li, Z. Shuai, and C. Wang, "Global stability of multigroup epidemic models with distributed delays," Journal of Mathematical Analysis and Applications, vol. 361, no. 1, pp. 3847, 2010.

[27] H. Shu, D. Fan, and J. Wei, "Global stability of multi-group SEIR epidemic models with distributed delays and nonlinear transmission," Nonlinear Analysis: Real World Applications, vol. 13, no. 4, pp. 1581-1592, 2012.

[28] C. Zhang, W. Li, and K. Wang, "Boundedness for network of stochastic coupled van der Pol oscillators with time-varying delayed coupling," Applied Mathematical Modelling, vol. 37, pp. 5394-5402, 2013.

[29] C. Zhang, W. Li, H. Su, and K. Wang, "A graph-theoretic approach to boundedness of stochasic Cohen-Grossberg neural networks with Markovian switching," Applied Mathematics and Computation, vol. 219, pp. 9165-9173, 2013.

[30] H. Su, W. Li, and K. Wang, "Global stability analysis of discretetime coupled systems on networks and its applications," Chaos, vol. 22, Article ID 033135, 2012.

[31] W. Li, H. Su, D. Wei, and K. Wang, "Global stability of coupled nonlinear systems with Markovian switching," Communications in Nonlinear Science and Numerical Simulation, vol. 17, no. 6, pp. 2609-2616, 2012.

[32] W. Li, H. Su, and K. Wang, "Global stability analysis for stochastic coupled systems on networks," Automatica, vol. 47, no. 1, pp. 215-220, 2011.

[33] W. Li, H. Song, Y. Qu, and K. Wang, "Global exponential stability for stochastic coupled systems on networks with Markovian switching," Systems and Control Letters, vol. 62, pp. 468-474, 2013.

[34] W. Li, L. Pang, H. Su, and K. Wang, "Global stability for discrete Cohen-Grossberg neural networks with finite and infinite delays," Applied Mathematics Letters, vol. 25, pp. 22462251, 2012.

[35] Q. Gan, "Adaptive synchronization of stochastic neural networks with mixed time delays and reaction-diffusion terms," Nonlinear Dynamics, vol. 69, no. 4, pp. 2207-2219, 2012.

[36] Q. Gan, "Global exponential synchronization of generalized stochastic neural networks with mixed time-varying delays and reaction-diffusion terms," Neurocomputing, vol. 89, pp. 96-105, 2012.

[37] H. Su, Y. Qu, S. Gao, H. Song, and K. Wang, "A model of feedback control system on network and its stability analysis," Communications in Nonlinear Science and Numerical Simulation, vol. 18, no. 7, pp. 1822-1831, 2013. 


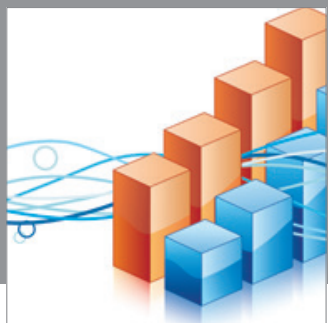

Advances in

Operations Research

mansans

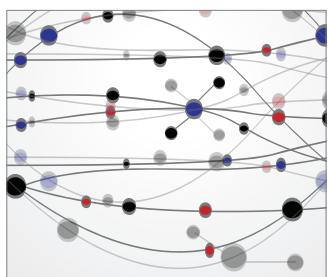

The Scientific World Journal
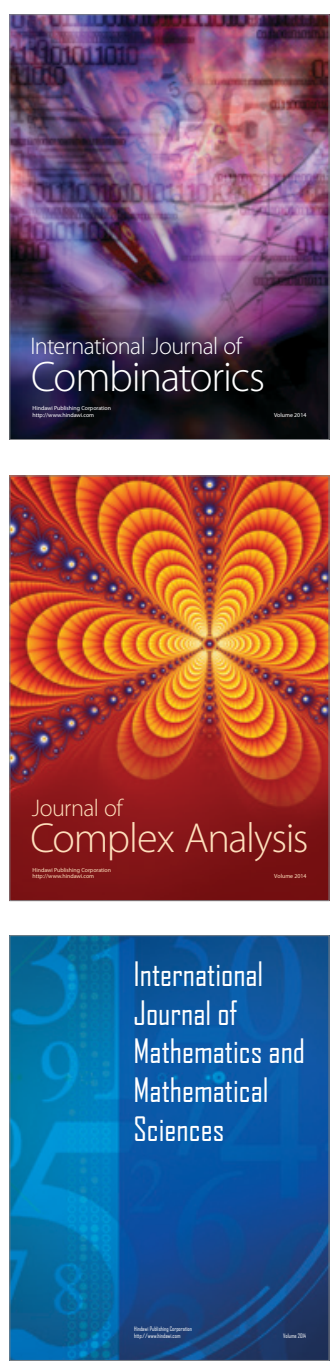
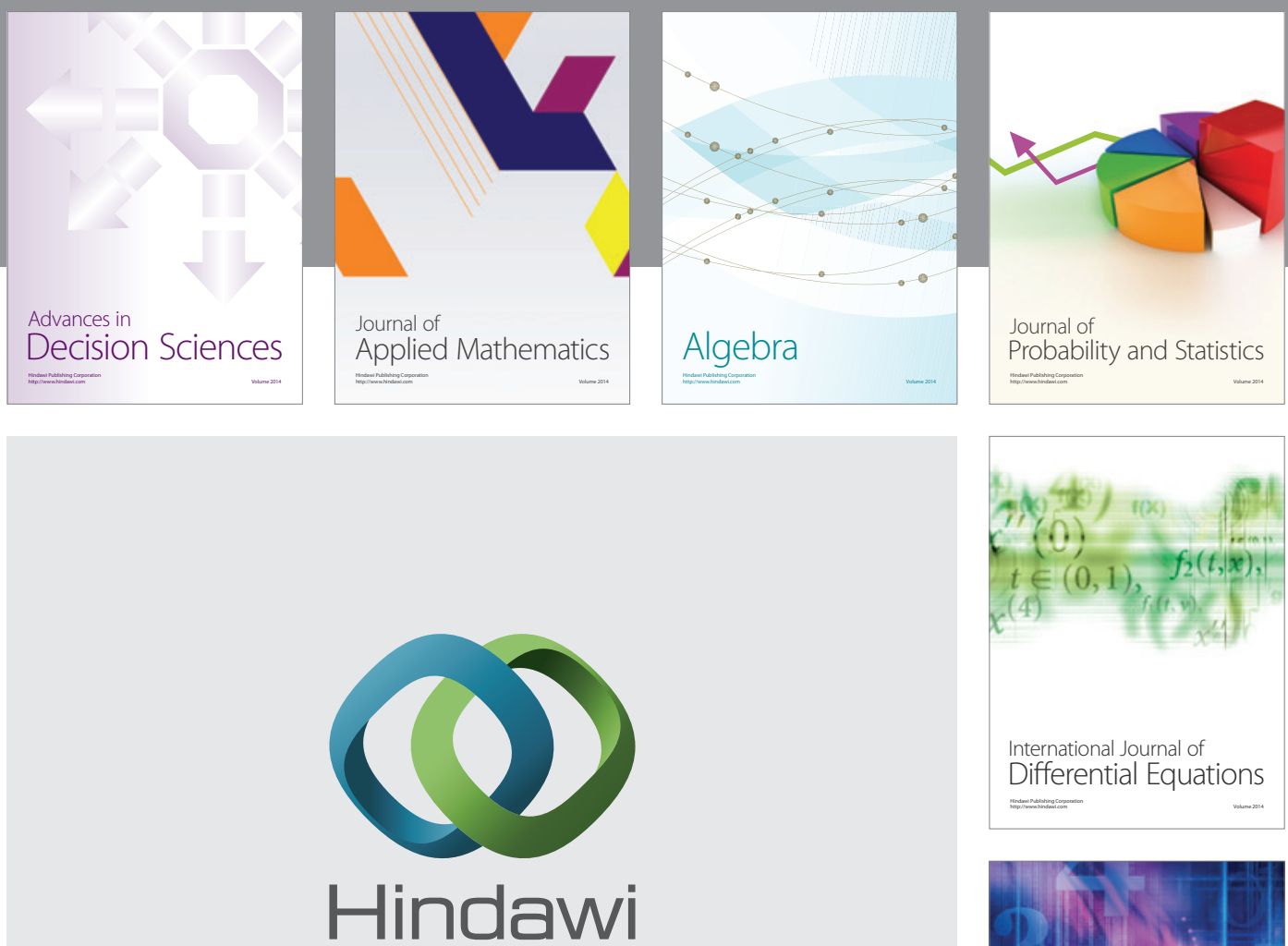

Submit your manuscripts at http://www.hindawi.com
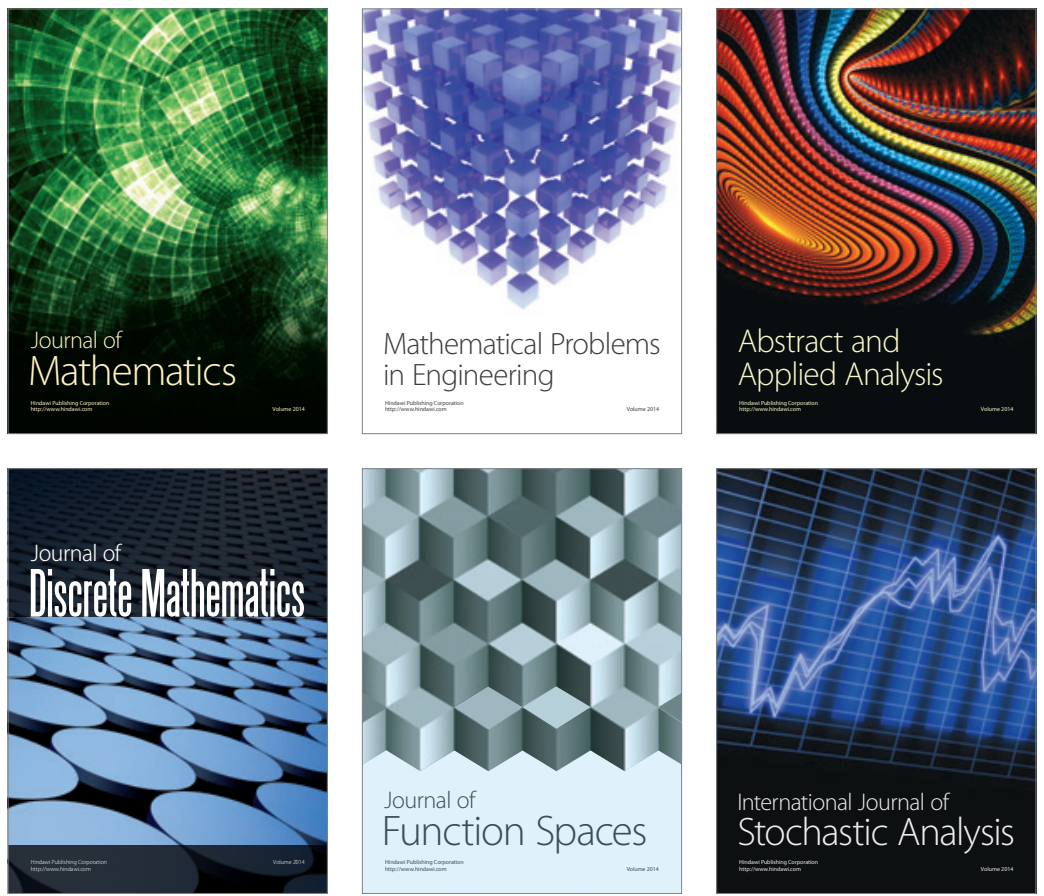

Journal of

Function Spaces

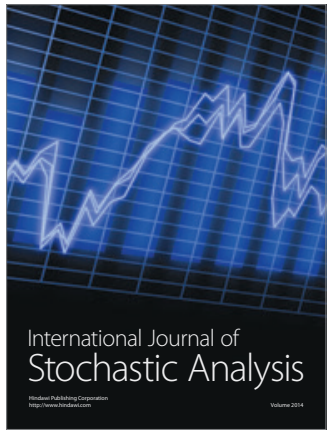

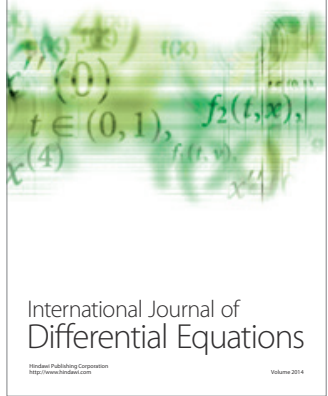
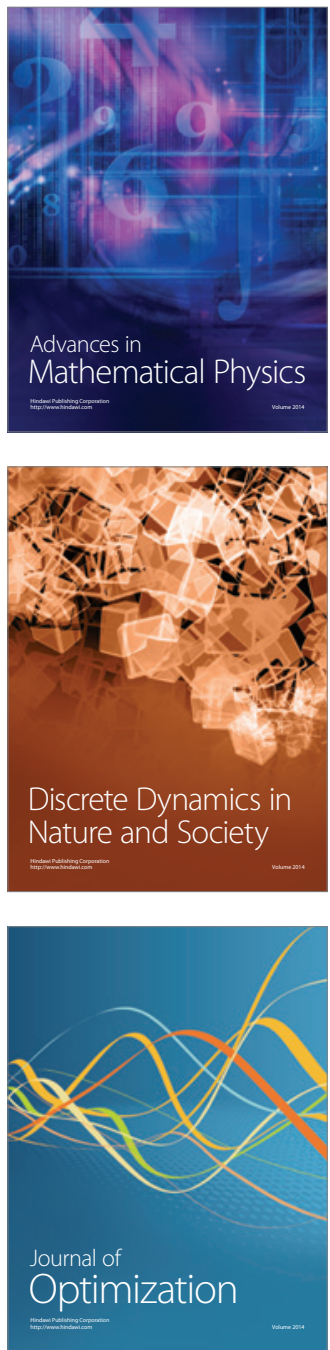